\title{
Optimal Preview Control for Discrete-Time Systems in Multirate Output Sampling
}

\author{
Fucheng Liao and Yujian Guo \\ School of Mathematics and Physics, University of Science and Technology Beijing, Beijing 100083, China \\ Correspondence should be addressed to Fucheng Liao; fcliao@ustb.edu.cn
}

Received 9 August 2016; Accepted 22 September 2016

Academic Editor: Michele Brun

Copyright ( 2016 F. Liao and Y. Guo. This is an open access article distributed under the Creative Commons Attribution License, which permits unrestricted use, distribution, and reproduction in any medium, provided the original work is properly cited.

\begin{abstract}
This paper studies the disturbance preview optimal control problem for discrete-time systems with multirate output sampling. By constructing the error system and using the discrete lifting technique, we reduce the multirate preview control problem to a singlerate one for a formal augmented system. Then, applying preview control theory, the optimal preview control law of the augmented error system is obtained. Meanwhile, we introduce a discrete integrator to eliminate the static error. Then we study a method to design a controller with preview action for the original system. And the existence conditions of the controller are also discussed in detail. Finally, numerical simulation is included to illustrate the effectiveness of the proposed method.
\end{abstract}

\section{Introduction}

In multirate output control systems, each component of the input vectors only changes once, and each component of the output vectors is detected several times during one sampling period. The increase in sampling frequency of the output vectors allows the controllers to obtain more information on the controlled objects and to acquire stronger control ability [1]. Reference [2] studied a "multirate output controller" and designed a multirate output feedback controller for a class of time-invariant continuous-time systems. References $[3,4]$ studied the design of the multirate output sampling functional observer and periodic output feedback controller based on fast output sampling, respectively. The above two papers take full advantage of the multirate output control systems' ability to increase the number of detecting output vectors.

Optimal control theory has numerous applications in both science and engineering. Recent reference [5] develops a comprehensive optimality theory of problems described by ordinary and partial differential inclusions and presents a number of new and important results. The theoretical basis of preview control is optimal control. Preview control theory takes full advantage of the known future reference signals or disturbance signals to improve the performance of control systems. Preview control theory has generated a whole set of theories and methods during its development [6-8]. There are many studies in the literature on this theory, and the perfect one is the study based on linear time-invariant systems [9]. Lately, progress has been made in combining preview control theory with stochastic systems [10], nonlinear systems [11], and descriptor systems [12]. Moreover, preview control theory has been applied successfully to vehicle active suspensions [13], wind turbines [14], and other aspects.

The sampling rate for control systems may be limited by physical hardware. For example, the sampling rate in the control of hard-disk systems is determined by the number of sectors on circular tracks and the spindle speed. A low sampling rate can reduce the cost of hardware resources. However, an overly low sampling rate has a bad effect on system performance and system stability. Generally, the performance of control systems can be improved by increasing sampling frequency properly [15]. In [16], it has been shown that there is an advantage in updating feedforward control input at a higher rate in track-seeking control. These cases motivate the research work for preview control theory of multirate systems.

Research into preview control theory in multirate settings began with [17]. In that paper, Liao et al. presented an LQ optimal preview servomechanism design method for linear discrete-time systems with time-delay in a multirate setting. The paper eliminated the time-delay by state augmentation, 
similar to the technique offered in [6]. Reference [18] discussed the optimal preview control problem for a class of multirate sampled systems with state time-delay. Reference [19] applied preview control theory to solve the optimal preview control problem for discrete-time descriptor causal systems in a multirate setting. But the above papers all study the preview control problem for multirate input control systems. Compared with the frequent changes of input vectors for multirate input control systems, multirate output control systems make the structures of systems relatively simpler and suitable for industrial process control. So this paper studies optimal preview control for discrete-time systems in multirate output sampling. We will use the following lemmas repeatedly in [20].

Lemma 1 (PBH rank test). $(A, B)$ is stabilizable if and only if, for any complex $\lambda$ satisfying $|\lambda| \geq 1$, the matrix $[\lambda I-A B]$ has full row rank. $(C, A)$ is detectable if and only if, for any complex $\lambda$ satisfying $|\lambda| \geq 1$, the matrix $\left[{ }_{C}^{\lambda I-A}\right]$ has full column rank.

\section{Problem Description and Related Assumptions}

Consider the following linear discrete-time system:

$$
\begin{aligned}
x(k+1) & =A x(k)+B u(k)+E w(k) \\
y(k) & =C x(k),
\end{aligned}
$$

where $x(k) \in R^{n}, u(k) \in R^{m}, y(k) \in R^{p}$, and $w(k) \in R^{l}$ represent the state vector, the control input vector, the output vector, and the disturbance vector, respectively. $A, B, C$, and $E$ are known constant matrices with appropriate dimensions.

For system (1), we introduce the following assumptions.

Assumption 2. $(A, B)$ is stabilizable.

Assumption 3. $(C, A)$ is detectable.

Assumption 4. The input vector $u(k)$ can be input only at $k=$ $i N(i=0,1,2, \ldots)$, where $N$ is a positive integer.

Remark 5. Assumptions 2 and 3 are basic assumptions of control systems. Assumption 4 makes the system multirate. That is, the input vector $u(k)$ can be input once during every $N$ sampling interval and the output vector $y(k)$ can be measured at every moment.

Assumption 6. $u(i N+q)=u(i N), q=0,1,2, \ldots, N-1 ; i=$ $0,1,2, \ldots$

Remark 7. Suppose the system has zero-order-hold; that is, $u(i N+1)=u(i N+2)=\cdots u(i N+N-1)=u(i N)(i=$ $0,1,2, \ldots)$.

Assumption 8. The matrix

$$
\Psi=\left[\begin{array}{cc}
A-I & B \\
C & 0
\end{array}\right]
$$

has full row rank.
Remark 9. Assumption 8 is one of the basic assumptions of preview control theory in [6].

We assume that the disturbance signal is previewable.

Assumption 10. The future values of the disturbance $w(k+$ $1), w(k+2), \ldots, w\left(k+M_{d}\right)$, as well as the present and past values, are available at time $k$, where $M_{d}$ is the preview length of the disturbance and $M_{d}=S_{d} N, S_{d}$ is a nonnegative integer.

Furthermore, we assume the following conditions, for simplicity of discussion.

Assumption 11. The disturbance $w(k)$ converges to zero as time $k$ goes to infinity. That is,

$$
\lim _{k \rightarrow \infty} w(k)=0 .
$$

We assume reference signal $r(k)$ is given by the step function

$$
r(k)= \begin{cases}r, & k \geq k_{0} \\ 0, & k<k_{0} .\end{cases}
$$

According to Assumptions 8 and 11, there always exist constant vectors $x(\infty)$ and $u(\infty)$ such that

$$
\begin{aligned}
x(\infty) & =A x(\infty)+B u(\infty) \\
r & =C x(\infty) .
\end{aligned}
$$

That is,

$$
\left[\begin{array}{cc}
A-I & B \\
C & 0
\end{array}\right]\left[\begin{array}{l}
x(\infty) \\
u(\infty)
\end{array}\right]=\left[\begin{array}{l}
0 \\
I
\end{array}\right] r .
$$

For the equation of $\left[\begin{array}{l}x(\infty) \\ u(\infty)\end{array}\right]$, the rank of the coefficient matrix is the same as that of the augmented matrix, by Assumption 8 . So, this equation has a solution. In general, we take one solution from the above equation and express this solution as

$$
\left[\begin{array}{l}
x(\infty) \\
u(\infty)
\end{array}\right]=\left[\begin{array}{cc}
A-I & B \\
C & 0
\end{array}\right]^{\dagger}\left[\begin{array}{l}
0 \\
I
\end{array}\right] r
$$

where $(\cdot)^{\dagger}$ denotes the right inverse.

Define the new vectors,

$$
\begin{aligned}
& \tilde{x}(k)=x(k)-x(\infty), \\
& \tilde{u}(k)=u(k)-u(\infty),
\end{aligned}
$$

and the error signal,

$$
e(k)=y(k)-r(k) .
$$

From (1) and (5), we get the error system

$$
\begin{aligned}
\tilde{x}(k+1) & =A \tilde{x}(k)+B \tilde{u}(k)+E w(k) \\
e(k) & =C \tilde{x}(k) .
\end{aligned}
$$


In this paper, we wish to design a type-1 servo controller such that the output $y(k)$ can track reference signal $r(k)$ without steady-state error. That is,

$$
\lim _{k \rightarrow \infty} e(k)=0
$$

To achieve good transient response, we introduce the quadratic performance index

$$
J=\sum_{k=0}^{\infty}\left[e^{T}(k) Q_{e} e(k)+\widetilde{u}^{T}(k) H_{u} \widetilde{u}(k)\right],
$$

where the weight matrices satisfy $Q_{e}>0, H_{u}>0$.

\section{The Derivation of the Augmented Error System}

In this section, we eliminate the multirate feature of Assumption 4 using the discrete lifting technique. Using the lifting scheme of this paper, we can construct the necessary augmented error system. Hence the preview control problem for a multirate output sampling system is converted into an optimal regulator problem for the normal augmented error system. And the controller with preview action for the augmented error system can be obtained by applying optimal preview control theory.

3.1. Discrete Lifting for the Multirate Output Sampling System. According to Assumptions 4 and 6 , the input vector $\widetilde{u}(k)$ of system (10) can be input at $k=i N(i=0,1,2, \ldots)$ and $\tilde{u}(i N+$ $q)=\widetilde{u}(i N)(q=0,1,2, \ldots, N-1)$.

Noticing the first equation in (10) and the characteristic of $\widetilde{u}(\cdot)$, we get

$$
\begin{gathered}
\tilde{x}(i N+1)=A \tilde{x}(i N)+B \tilde{u}(i N)+E w(i N) \\
\tilde{x}(i N+2)=A \widetilde{x}(i N+1)+B \widetilde{u}(i N)+E w(i N+1) \\
\vdots \\
\tilde{x}(i N+N)=A \tilde{x}(i N+N-1)+B \widetilde{u}(i N) \\
\quad+E w(i N+N-1) .
\end{gathered}
$$

That is,

$$
\begin{aligned}
& {\left[\begin{array}{c}
\tilde{x}(i N+1) \\
\tilde{x}(i N+2) \\
\vdots \\
\tilde{x}(i N+N)
\end{array}\right]} \\
& =\left[\begin{array}{llll}
A & & & \\
& A & & \\
& & \ddots & \\
& & & A
\end{array}\right]\left[\begin{array}{c}
\tilde{x}(i N) \\
\tilde{x}(i N+1) \\
\tilde{x}(i N+N-1)
\end{array}\right]
\end{aligned}
$$

Similarly, we obtain the equation of the error signal $e(k)$

$$
\begin{gathered}
{\left[\begin{array}{c}
e(i N) \\
e(i N+1) \\
e(i N+N-1)
\end{array}\right]} \\
=\left[\begin{array}{ccc}
C & & \\
& C & \\
& & \ddots \\
& & C
\end{array}\right]\left[\begin{array}{c}
\tilde{x}(i N) \\
\tilde{x}(i N+1) \\
\vdots \\
\tilde{x}(i N+N-1)
\end{array}\right] .
\end{gathered}
$$

We introduce the vectors as follows:

$$
\hat{x}(i N)=\left[\begin{array}{c}
\tilde{x}(i N) \\
\tilde{x}(i N+1) \\
\vdots \\
\tilde{x}(i N+N-1)
\end{array}\right] \in R^{N n},
$$$$
\widehat{u}(i N)=\left[\begin{array}{c}
\tilde{u}(i N) \\
\tilde{u}(i N) \\
\vdots \\
\tilde{u}(i N)
\end{array}\right] \in R^{N m},
$$$$
E(i N)=\left[\begin{array}{c}
e(i N) \\
e(i N+1) \\
\vdots \\
e(i N+N-1)
\end{array}\right] \in R^{N p},
$$

$$
W(i N)=\left[\begin{array}{c}
w(i N) \\
w(i N+1) \\
\vdots \\
w(i N+N-1)
\end{array}\right] \in R^{N l},
$$




$$
\begin{aligned}
& \widehat{A}=\left[\begin{array}{llll}
A & & & \\
& A & & \\
& & \ddots & \\
& & & A
\end{array}\right], \\
& \widehat{B}=\left[\begin{array}{llll}
B & & & \\
& B & & \\
& & \ddots & \\
& & & \\
& & & B
\end{array}\right], \\
& \widehat{C}=\left[\begin{array}{llll}
C & & & \\
& C & & \\
& & \ddots & \\
& & & C
\end{array}\right] \text {, } \\
& \widehat{E}=\left[\begin{array}{llll}
E & & & \\
& E & & \\
& & \ddots & \\
& & & E
\end{array}\right] .
\end{aligned}
$$

Combining (14) and (15), we get

$$
\begin{aligned}
\widehat{x}(i N+1) & =\widehat{A} \widehat{x}(i N)+\widehat{B} \widehat{u}(i N)+\widehat{E} W(i N) \\
E(i N) & =\widehat{C} \widehat{x}(i N) .
\end{aligned}
$$

Now we have successfully transformed the multirate output sampling system into the single-rate system by the lifting technique.

3.2. Introduction of Integral Compensation. To achieve the robust servo property mentioned in the previous section and eliminate the steady-state error, we need to introduce the discrete integrator, defined by

$$
V(i N+1)=V(i N)+E(i N),
$$

where

$$
V(i N)=\left[\begin{array}{c}
v(i N) \\
v(i N+1) \\
\vdots \\
v(i N+N-1)
\end{array}\right] .
$$

Equation (18) is the discrete integrator and we can derive the following equation:

$$
\begin{gathered}
{\left[\begin{array}{c}
v(i N) \\
v(i N+1) \\
\vdots \\
v(i N+N-1)
\end{array}\right]} \\
=\left[\begin{array}{c}
v(0)+\sum_{s=0}^{i N-1} e(s) \\
v(1)+\sum_{s=0}^{i N-1} e(s+1) \\
\vdots \\
v(N-1)+\sum_{s=0}^{i N-1} e(s+N-1)
\end{array}\right] .
\end{gathered}
$$

That is,

$$
V(i N)=V(0)+\sum_{s=0}^{i N-1} E(s) .
$$

Noticing the second equation in (17), we obtain

$$
V(i N+1)=V(i N)+\widehat{C} \hat{x}(i N) .
$$

If the closed-loop system is stabilized, there exists a stationary value of $V(k)$. Let $V(\infty)=\lim _{k \rightarrow \infty} V(k)$ and define

$$
\widetilde{V}(k)=V(k)-V(\infty) .
$$

From (22), we have

$$
\widetilde{V}(i N+1)=\widetilde{V}(i N)+\widehat{C} \hat{x}(i N) .
$$

This enables us to add a degree of freedom to improve the transient response of the closed-loop system since $V(\infty)$ can be arbitrarily assigned [17].

Combining (17) and (24) and matching the corresponding block, we obtain the augmented system

$$
\begin{aligned}
X_{0}(i N+1) & =A_{0} X_{0}(i N)+B_{0} \widehat{u}(i N)+E_{0} W(i N) \\
E(i N) & =C_{0} X_{0}(i N),
\end{aligned}
$$

where

$$
\begin{aligned}
& X_{0}(i N)=\left[\begin{array}{c}
\bar{x}(i N) \\
\bar{x}(i N+1) \\
\vdots \\
\bar{x}(i N+N-1)
\end{array}\right] \text {, } \\
& A_{0}=\left[\begin{array}{llll}
\bar{A} & & & \\
& \bar{A} & & \\
& & \ddots & \\
& & \bar{A}
\end{array}\right] \text {, }
\end{aligned}
$$




$$
\begin{aligned}
& B_{0}=\left[\begin{array}{llll}
\bar{B} & & & \\
& \bar{B} & & \\
& & \ddots & \\
& & & \bar{B}
\end{array}\right] \text {, } \\
& E_{0}=\left[\begin{array}{llll}
\bar{E} & & & \\
& \bar{E} & & \\
& & \ddots & \\
& & & \bar{E}
\end{array}\right] \text {, } \\
& C_{0}=\left[\begin{array}{llll}
\bar{C} & & & \\
& \bar{C} & & \\
& & \ddots & \\
& & & \bar{C}
\end{array}\right] \text {, } \\
& \bar{x}(i N)=\left[\begin{array}{l}
\widetilde{x}(i N) \\
\widetilde{v}(i N)
\end{array}\right], \\
& \bar{A}=\left[\begin{array}{ll}
A & 0 \\
C & I
\end{array}\right], \\
& \bar{B}=\left[\begin{array}{l}
B \\
0
\end{array}\right] \text {, } \\
& \bar{E}=\left[\begin{array}{l}
E \\
0
\end{array}\right], \\
& \bar{C}=\left[\begin{array}{ll}
C & 0
\end{array}\right] \text {. }
\end{aligned}
$$

Augmented system (25) is the formal system for designing the controller.

3.3. Modification of the Performance Index Function. From (12) and the structure of $E(i N)$ and $\widehat{u}(i N)$, we can see

$$
\begin{aligned}
J & =\sum_{k=0}^{\infty}\left[e^{T}(k) Q_{e} e(k)+\widetilde{u}^{T}(k) H_{u} \widetilde{u}(k)\right] \\
& =\sum_{i=0}^{\infty} \sum_{j=0}^{N-1}\left[e^{T}(i N+j) Q_{e} e(i N+j)\right. \\
& \left.+\widetilde{u}^{T}(i N+j) H_{u} \widetilde{u}(i N+j)\right]=\sum_{i=0}^{\infty}\left[E^{T}(i N)\right. \\
& \left.\cdot \widehat{Q}_{e} E(i N)+\widehat{u}^{T}(i N) \widehat{H}_{u} \widehat{u}(i N)\right]
\end{aligned}
$$

where $\widehat{Q}_{e}=\operatorname{diag}(\underbrace{Q_{e} Q_{e} \cdots Q_{e}}_{N}), \widehat{H}_{u}=$ $\operatorname{diag}(\underbrace{H_{u}^{H_{u}} H_{u} \cdots H_{u}}_{N})$, and $\widehat{Q}_{e} \in R^{(N p) \times(N p)}, \widehat{Q}_{e}>0$, $\widehat{H}_{u} \in R^{(N m) \times(N m)}, \widehat{H}_{u}>0$.
Noticing the second equation of (17), the performance index (27) can be rewritten as

$$
\begin{aligned}
J= & \sum_{i=0}^{\infty}\left[\widehat{x}^{T}(i N)\left(\widehat{C}^{T} \widehat{Q}_{e} \widehat{C}\right) \widehat{x}(i N)\right. \\
& \left.+\widehat{u}^{T}(i N) \widehat{H}_{u} \widehat{u}(i N)\right] .
\end{aligned}
$$

In order to regularize the LQ optimal control problem and guarantee the existence of a stabilizing solution to the associated Riccati equation, we modify the performance index as follows:

$$
\begin{aligned}
\widehat{J}= & J+\sum_{i=0}^{\infty} \widetilde{V}^{T}(i N) \widehat{Q}_{\nu} \widetilde{V}(i N) \\
& =\sum_{i=0}^{\infty}\left[\widehat{x}^{T}(i N)\left(\widehat{C}^{T} \widehat{Q}_{e} \widehat{C}\right) \widehat{x}(i N)\right. \\
& \left.+\widehat{u}^{T}(i N) \widehat{H}_{u} \widehat{u}(i N)+\widetilde{V}^{T}(i N) \widehat{Q}_{\nu} \widetilde{V}(i N)\right] \\
& =\sum_{i=0}^{\infty}\left[X_{0}^{T}(i N) \widehat{Q}_{0} X_{0}(i N)+\widehat{u}^{T}(i N) \widehat{H}_{u} \widehat{u}(i N)\right],
\end{aligned}
$$

where $Q_{v}>0, \widehat{Q}_{v}=\operatorname{diag}(\underbrace{Q_{v} Q_{v} \cdots Q_{v}}), \widehat{Q}_{0}=$ $\operatorname{diag}(\underbrace{\bar{Q} \bar{Q} \cdots \bar{Q}}_{N})$, and $\bar{Q}=\left[\begin{array}{lll}C^{T} Q_{e} C & \\ & & \\ & Q_{v}\end{array}\right]$.

Now, the preview control problem of multirate output control system (1) becomes a standard LQ optimal preview control problem of augmented system (25) with performance index (29).

Applying the results in [6-8], we can derive the following theorem.

Theorem 12. If $\left(A_{0}, B_{0}\right)$ is stabilizable and $\left(\widehat{Q}_{0}^{1 / 2}, A_{0}\right)$ is detectable, the optimal preview controller of system (25) minimizing the performance index (29) is given by

$$
\widehat{u}(i N)=\widehat{F} X_{0}(i N)+\sum_{j=0}^{S_{d}-1} F_{d}(j) W((i+j) N),
$$

where

$$
\begin{aligned}
\widehat{F} & =-\left[\widehat{H}_{u}+B_{0}^{T} \widehat{P} B_{0}\right]^{-1} B_{0}^{T} \widehat{P} A_{0} \\
F_{d}(j) & =-\left[\widehat{H}_{u}+B_{0}^{T} \widehat{P} B_{0}\right]^{-1} B_{0}^{T}\left(F^{T}{ }_{c}\right)^{j} \widehat{P} E_{0} \\
& \quad\left(j=0,1, \ldots, S_{d}-1\right) \\
F_{c} & =A_{0}+B_{0} \widehat{F} \\
& =A_{0}-B_{0}\left[\widehat{H}_{u}+B_{0}^{T} \widehat{P} B_{0}\right]^{-1} B_{0}^{T} \widehat{P} A_{0},
\end{aligned}
$$

and $\widehat{P}$ is the unique symmetric semi-positive definite solution of the algebraic Riccati equation,

$$
\widehat{P}=A_{0}^{T} \widehat{P} A_{0}-A_{0}^{T} \widehat{P} B_{0}\left[\widehat{H}_{u}+B_{0}^{T} \widehat{P} B_{0}\right]^{-1} B_{0}^{T} \widehat{P} A_{0}+\widehat{Q}_{0} .
$$


3.4. Realization of the System. In the processes of constructing the augmented system, the order of formal system (25) increases highly. As a result, the order of corresponding Riccati equation (32) is very high. We study a method to solve high-order Riccati equation (32) by solving a low-order Riccati equation. Noticing the structure of the coefficient matrices $A_{0}$ and $B_{0}$ for system (25) and the weight matrices $\widehat{Q}_{0}$ and $\widehat{H}_{u}$ for performance index (29), the solution of the Riccati equation (32) can be decomposed into

$$
\widehat{P}=\left[\begin{array}{llll}
P & & & \\
& P & & \\
& & \ddots & \\
& & & P
\end{array}\right] .
$$

Substituting the partitioned matrix of $\widehat{P}$ into (32), we derive

$$
P=\bar{A}^{T} P \bar{A}-\bar{A}^{T} P \bar{B}\left[H_{u}+\bar{B}^{T} P \bar{B}\right]^{-1} \bar{B}^{T} P \bar{A}+\bar{Q} .
$$

In fact, we can obtain (32) by repeating (34) $N$ times. Thus, the solution of high-order Riccati equation (32) is transformed into solving low-order Riccati equation (34).

And this is equivalent to solving (34) for the following system:

$$
\bar{x}(i N+1)=\bar{A} \bar{x}(i N)+\bar{B} \widetilde{u}(i N)+\bar{E} w(i N) .
$$

That is,

$$
\begin{aligned}
{\left[\begin{array}{l}
\tilde{x}(i N+1) \\
\widetilde{v}(i N+1)
\end{array}\right]=} & {\left[\begin{array}{ll}
A & 0 \\
C & I
\end{array}\right]\left[\begin{array}{l}
\widetilde{x}(i N) \\
\widetilde{v}(i N)
\end{array}\right]+\left[\begin{array}{l}
B \\
0
\end{array}\right] \widetilde{u}(i N) } \\
& +\left[\begin{array}{l}
E \\
0
\end{array}\right] w(i N) .
\end{aligned}
$$

We know that (34) has a unique symmetric semi-positive definite solution if and only if $\left(\bar{Q}^{1 / 2}, \bar{A}\right)$ is detectable. Notice that (25) can be written as

$$
\begin{aligned}
& {\left[\begin{array}{l}
\tilde{x}(i N+1) \\
\widetilde{v}(i N+1)
\end{array}\right]=\left[\begin{array}{ll}
A & 0 \\
C & I
\end{array}\right]\left[\begin{array}{l}
\tilde{x}(i N) \\
\tilde{v}(i N)
\end{array}\right]+\left[\begin{array}{l}
B \\
0
\end{array}\right] \tilde{u}(i N)} \\
& +\left[\begin{array}{l}
E \\
0
\end{array}\right] w(i N) \\
& {\left[\begin{array}{l}
\tilde{x}(i N+2) \\
\tilde{v}(i N+2)
\end{array}\right]=\left[\begin{array}{ll}
A & 0 \\
C & I
\end{array}\right]\left[\begin{array}{l}
\tilde{x}(i N+1) \\
\tilde{v}(i N+1)
\end{array}\right]+\left[\begin{array}{l}
B \\
0
\end{array}\right] \tilde{u}(i N)} \\
& +\left[\begin{array}{l}
E \\
0
\end{array}\right] w(i N+1) \\
& {\left[\begin{array}{l}
\tilde{x}(i N+N) \\
\widetilde{v}(i N+N)
\end{array}\right]=\left[\begin{array}{ll}
A \\
C
\end{array}\right]\left[\begin{array}{l}
\tilde{x}(i N+N-1) \\
\widetilde{v}(i N+N-1)
\end{array}\right]} \\
& +\left[\begin{array}{l}
B \\
0
\end{array}\right] \widetilde{u}(i N)+\left[\begin{array}{l}
E \\
0
\end{array}\right] w(i N+N-1), \\
& \vdots
\end{aligned}
$$

and the first equation of (37) is (36). We can obtain $\tilde{u}(i N)$ by the solution of the Riccati equation (34) and the state vector $\left[\begin{array}{l}\widetilde{x}(i N) \\ \widetilde{v}(i N)\end{array}\right]$. Substituting $\widetilde{u}(i N)$ into the first equation of (37), $\left[\begin{array}{l}\tilde{x}(i N+1) \\ \widetilde{v}(i N+1)\end{array}\right]$ is obtained. Then, substituting $\left[\begin{array}{l}\tilde{x}(i N+1) \\ \widetilde{v}(i N+1)\end{array}\right]$ and $\widetilde{u}(i N)$ into the second equation of $(37),\left[\begin{array}{l}\widetilde{x}(i N+2) \\ \widetilde{v}(i N+2)\end{array}\right]$ is obtained. By that analogy, $\left[\begin{array}{l}\tilde{x}(i N+N) \\ \widetilde{v}(i N+N)\end{array}\right]$ is obtained. Then, the simulation can be realized.

\section{Existence Conditions of the Controller}

It is a well-known fact that the stabilizability of $\left(A_{0}, B_{0}\right)$ guarantees that the state feedback gain in Theorem 12 exists. And the detectability of $\left(\widehat{Q}_{0}^{1 / 2}, A_{0}\right)$ ensures that Riccati equation (32) has a unique symmetric semi-positive solution [20].

First, we examine the stabilizability of $\left(A_{0}, B_{0}\right)$.

Lemma 13. $\left(A_{0}, B_{0}\right)$ is stabilizable if and only if $(\bar{A}, \bar{B})$ is stabilizable.

Proof. By the $\mathrm{PBH}$ rank test,

$$
\begin{aligned}
& A_{0}=\operatorname{diag}(\underbrace{\bar{A} \bar{A} \cdots \bar{A}}_{N}), \\
& B_{0}=\operatorname{diag}(\underbrace{\bar{B} \bar{B} \cdots \bar{B}}_{N}),
\end{aligned}
$$

for any complex $\lambda$, if $|\lambda| \geq 1$, we have

$$
\begin{aligned}
& \operatorname{rank}[\widehat{A}-\lambda I \mid \widehat{B}] \\
& =\operatorname{rank}\left[\begin{array}{cccc|ccc}
\bar{A}-\lambda I & & & & \bar{B} & & \\
& \bar{A}-\lambda I & & & \bar{B} & & \\
& & \ddots & & & & \\
& & & \bar{A}-\lambda I & & & \bar{B}
\end{array}\right] \\
& =\operatorname{rank}\left[\begin{array}{llll}
{[\bar{A}-\lambda I \mid \bar{B}]} & & & \\
& {[\bar{A}-\lambda I \mid \bar{B}]} & & \\
& & \ddots & \\
& & {[\bar{A}-\lambda I \mid \bar{B}]}
\end{array}\right] \\
& =N \cdot \operatorname{rank}[\bar{A}-\lambda I \mid \bar{B}] \text {. }
\end{aligned}
$$

Thus, matrix $[\widehat{A}-\lambda I \mid \widehat{B}]$ has full row rank if and only if matrix $[\bar{A}-\lambda I \mid \bar{B}]$ has full row rank. So, Lemma 13 is established.

Lemma 14. $(\bar{A}, \bar{B})$ is stabilizable if and only if $(A, B)$ is stabilizable and the matrix $\left[\begin{array}{cc}A-I & B \\ C & 0\end{array}\right]$ has full row rank.

Noticing the structure of $\bar{A}$ and $\bar{B}$, one gets this lemma in [6].

Therefore, one obtains the following theorem on the stabilizability of $\left(A_{0}, B_{0}\right)$.

Theorem 15. $\left(A_{0}, B_{0}\right)$ is stabilizable if and only if $(A, B)$ is stabilizable and the matrix $\left[\begin{array}{cc}A-I & B \\ C & 0\end{array}\right]$ has full row rank. 
Theorem 15 guarantees that $\left(A_{0}, B_{0}\right)$ is stabilizable under Assumptions 2 and 8.

Next, we discuss the detectability of $\left(\widehat{Q}_{0}^{1 / 2}, A_{0}\right)$.

Lemma 16. $\left(\widehat{Q}_{0}^{1 / 2}, A_{0}\right)$ is detectable if and only if $\left(\bar{Q}^{1 / 2}, \bar{A}\right)$ is detectable.

Noticing the structure of $A_{0}=\operatorname{diag}(\underbrace{\bar{A} \bar{A} \cdots \bar{A}}_{N})$ and $\widehat{Q}_{0}=\operatorname{diag}(\underbrace{\bar{Q} \bar{Q} \cdots \bar{Q}}_{N})$, one can prove Lemma 16 by using the similar method in Lemma 13. Here, one omits the proof.

Lemma 17. $\left(\bar{Q}^{1 / 2}, \bar{A}\right)$ is detectable if and only if $(C, A)$ is detectable and $Q_{v}>0$.

Proof. According to [17], $\left(\bar{Q}^{1 / 2}, \bar{A}\right)$ is detectable if and only if $\left(Q_{e}^{1 / 2} C, A\right)$ is detectable and $Q_{v}>0$. And $Q_{e}>0$, so the detectability of $\left(Q_{e}^{1 / 2} C, A\right)$ is equivalent to that of $(C, A)$. Then Lemma 17 holds.

From Lemmas 16 and 17, we obtain.

Theorem 18. $\left(\widehat{Q}_{0}^{1 / 2}, A_{0}\right)$ is detectable if and only if $(C, A)$ is detectable and $Q_{v}>0$.

Consequently, we see from Theorems 15 and 18 that the solvability of the Riccati equation (32) is guaranteed under the standard servomechanism assumptions for the original system.

\section{Design of an Optimal Preview Controller for the Original System}

Now we return to the controller design with preview action for the original system. We can see that Riccati equation (32) can be obtained by repeating (34) $N$ times, as pointed out above. Then we consider the optimal input (30) of system (25) and associated (31) and (32) in Theorem 12.

Noticing the structure of $\widehat{P}, \widehat{H}_{u}$, and $B_{0}$ and calculating $\widehat{H}_{u}+B_{0}{ }^{T} \widehat{P} B_{0}$, we have

$$
\begin{aligned}
& \widehat{H}_{u}+B_{0}^{T} \widehat{P} B_{0}=\left[\begin{array}{llll}
H_{u} & & & \\
& H_{u} & & \\
& & \ddots & \\
& & & H_{u}
\end{array}\right] \\
& +\left[\begin{array}{cccc}
\bar{B} & & & \\
& \bar{B} & & \\
& & \ddots & \\
& & & \bar{B}
\end{array}\right]^{T}\left[\begin{array}{llll}
P & & & \\
& P & & \\
& & \ddots & \\
& & & P
\end{array}\right]\left[\begin{array}{llll}
\bar{B} & & & \\
& \bar{B} & \\
& & \ddots & \\
& & & \bar{B}
\end{array}\right] \\
& =\left[\begin{array}{cccc}
H_{u}+\bar{B}^{T} P \bar{B} & & & \\
& H_{u}+\bar{B}^{T} P \bar{B} & & \\
& & \ddots & \\
& & H_{u}+\bar{B}^{T} P \bar{B}
\end{array}\right] \text {. }
\end{aligned}
$$

This is the block diagonal matrix. Similarly, we notice that $\widehat{F}, F_{d}(j)$, and $F_{c}$ are all block diagonal matrices and the submatrix blocks of the diagonal are identical. Calculating these block diagonal matrices and submitting them into (30) we get

$$
\widetilde{u}(i N)=F \bar{x}(i N)+\sum_{j=0}^{S_{d}-1} M_{d}(j) w((i+j) N),
$$

where

$$
\begin{gathered}
F=-\left[H_{u}+\bar{B}^{T} P \bar{B}\right]^{-1} \bar{B}^{T} P \bar{A} \\
M_{d}(j)=-\left[H_{u}+\bar{B}^{T} P \bar{B}\right]^{-1} \bar{B}^{T}\left(M_{c}^{T}\right)^{j} P \bar{E} \\
\quad\left(j=0,1, \ldots, S_{d}-1\right) \\
M_{c}=\bar{A}+\bar{B} F=\bar{A}-\bar{B}\left[H_{u}+\bar{B}^{T} P \bar{B}\right]^{-1} \bar{B}^{T} P \bar{A}
\end{gathered}
$$

and $P$ is the unique symmetric semi-positive definite solution of algebraic Riccati equation (34).

We decompose $F$ into

$$
F=\left[\begin{array}{ll}
F_{x} & F_{v}
\end{array}\right] .
$$

Equation (41) can be written as

$$
\begin{aligned}
\widetilde{u}(i N)= & {\left[\begin{array}{ll}
F_{x} & F_{v}
\end{array}\right]\left[\begin{array}{l}
\tilde{x}(i N) \\
\widetilde{v}(i N)
\end{array}\right] } \\
& +\sum_{j=0}^{S_{d}-1} M_{d}(j) w((i+j) N) .
\end{aligned}
$$

That is,

$$
\begin{aligned}
\widetilde{u}(i N)= & F_{x} \widetilde{x}(i N)+F_{v} \widetilde{v}(i N) \\
& +\sum_{j=0}^{S_{d}-1} M_{d}(j) w((i+j) N) .
\end{aligned}
$$

Further, the above equation can be written as

$$
\begin{aligned}
u(i N)-u(\infty)= & F_{x}[x(i N)-x(\infty)] \\
& +F_{v}[v(i N)-v(\infty)] \\
& +\sum_{j=0}^{S_{d}-1} M_{d}(j) w((i+j) N) .
\end{aligned}
$$

From (21), we have $v(i N)=v(0)+\sum_{s=0}^{i N-1} e(s)$; that is,

$$
\begin{aligned}
u(i N)= & F_{x} x(i N)+F_{v} \sum_{s=0}^{i N-1} e(s) \\
& +\sum_{j=0}^{S_{d}-1} M_{d}(j) w((i+j) N)-F_{x} x(\infty) \\
& +u(\infty)+F_{v}(v(0)-v(\infty)) .
\end{aligned}
$$


By substituting (7) into the above equation, we have

$$
\begin{aligned}
-F_{x} x(\infty)+u(\infty) & =\left[\begin{array}{ll}
-F_{x} & I
\end{array}\right]\left[\begin{array}{l}
x(\infty) \\
u(\infty)
\end{array}\right] \\
& =\left[\begin{array}{ll}
-F_{x} & I
\end{array}\right]\left[\begin{array}{cc}
A-I & B \\
C & 0
\end{array}\right]^{\dagger}\left[\begin{array}{l}
0 \\
I
\end{array}\right] r .
\end{aligned}
$$

Then we obtain the main theorem of this paper.

Theorem 19. If the following conditions hold:

(1) $(A, B)$ is stabilizable (Assumption 2 holds);

(2) $(C, A)$ is detectable (Assumption 3 holds);

(3) the matrix $\left[\begin{array}{cc}A-I & B \\ C & 0\end{array}\right]$ has full row rank (Assumption 8 holds);

(4) $Q_{e}>0, H_{u}>0, Q_{v}>0$.

Riccati equation (32) has a unique symmetric semi-positive definite solution, and the optimal control input of system (1) with multirate output sampling is

$$
\begin{aligned}
u(i N)= & F_{x} x(i N)+F_{v} \sum_{s=0}^{i N-1} e(s) \\
& +\sum_{j=0}^{S_{d}-1} M_{d}(j) w((i+j) N)+\Gamma r \\
& +F_{v}(v(0)-v(\infty)),
\end{aligned}
$$

where $F_{x}, F_{v}$, and $M_{d}(j)$ are determined by (42) and (43) and where

$$
\begin{aligned}
& \Gamma=\left[\begin{array}{ll}
-F_{x} & I
\end{array}\right]\left[\begin{array}{cr}
A-I & B \\
C & 0
\end{array}\right]^{\dagger}\left[\begin{array}{l}
0 \\
I
\end{array}\right] \\
& u(i N+j)=u(i N), j=1,2, \ldots, N-1 . \\
& \text { The closed-loop system of }(1) \text { is } \\
& x(i N+1)=A x(i N)+B u(i N)+E w(i N) \\
& x(i N+2)=A x(i N+1)+B u(i N)+E w(i N+1) \\
& \quad \vdots \\
& x(i N+N)=A x(i N+N-1)+B u(i N) \\
& +E w(i N+N-1) \\
& u(i N)=F_{x} x(i N)+F_{v} \sum_{s=0}^{i N-1} e(s) \\
& +\sum_{d=0} M_{d}(j) w((i+j) N)+\Gamma r \\
& +F_{v}(v(0)-v(\infty))
\end{aligned}
$$

$$
(i=0,1,2, \ldots) \text {. }
$$

\section{Numerical Example}

Consider a linear discrete-time system:

$$
\begin{aligned}
x(k+1)= & {\left[\begin{array}{cc}
-1.2 & -0.79 \\
0.6 & 0.15
\end{array}\right] x(k)+\left[\begin{array}{c}
1.51 \\
2.1
\end{array}\right] u(k) } \\
& +\left[\begin{array}{c}
-0.3 \\
0.23
\end{array}\right] w(k) \\
y(k)= & {[-3.3-2.1] x(k), }
\end{aligned}
$$

where the coefficient matrices are

$$
\begin{aligned}
& A=\left[\begin{array}{cc}
-1.2 & -0.79 \\
0.6 & 0.15
\end{array}\right], \\
& B=\left[\begin{array}{c}
1.51 \\
2.1
\end{array}\right], \\
& C=\left[\begin{array}{ll}
-3.3 & -2.1
\end{array}\right], \\
& E=\left[\begin{array}{l}
-0.3 \\
0.23
\end{array}\right] .
\end{aligned}
$$

Let $N=2$; that is, the input vector $u(k)$ can be input only at $k=2 i(i=0,1,2, \ldots)$. Let the initial conditions be $x(0)=$ $x(1)=\left[\begin{array}{l}0 \\ 0\end{array}\right]$ and $v(0)=26$. Take the weight matrices $Q_{e}=5$, $H_{u}=1$, and $Q_{v}=0.1$.

Through verifying, system (52) satisfies Assumptions 2, 3, and 8 . In this case, $\bar{A}, \bar{B}$, and $\bar{Q}$ are calculated to be

$$
\begin{aligned}
& \bar{A}=\left[\begin{array}{ccc}
-1.20 & -0.79 & 0 \\
0.60 & 0.15 & 0 \\
-3.30 & -2.10 & 1.00
\end{array}\right], \\
& \bar{B}=\left[\begin{array}{c}
1.51 \\
2.10 \\
0
\end{array}\right], \\
& \bar{Q}=\left[\begin{array}{ccc}
54.45 & 34.65 & 0 \\
34.65 & 22.05 & 0 \\
0 & 0 & 0.10
\end{array}\right] .
\end{aligned}
$$

Riccati equation (34) is

$$
\begin{aligned}
P= & {\left[\begin{array}{ccc}
-1.20 & -0.79 & 0 \\
0.60 & 0.15 & 0 \\
-3.30 & -2.10 & 1.00
\end{array}\right]^{T} P\left[\begin{array}{ccc}
-1.20 & -0.79 & 0 \\
0.60 & 0.15 & 0 \\
-3.30 & -2.10 & 1.00
\end{array}\right] } \\
& -\left[\begin{array}{ccc}
-1.20 & -0.79 & 0 \\
0.60 & 0.15 & 0 \\
-3.30 & -2.10 & 1.00
\end{array}\right]^{T}
\end{aligned}
$$




$$
\begin{aligned}
& \cdot P\left[\begin{array}{c}
1.51 \\
2.10 \\
0
\end{array}\right]\left[1+\left[\begin{array}{c}
1.51 \\
2.10 \\
0
\end{array}\right]^{T} P\left[\begin{array}{c}
1.51 \\
2.10 \\
0
\end{array}\right]\right]^{-1} \\
& \cdot\left[\begin{array}{c}
1.51 \\
2.10 \\
0
\end{array}\right]^{T} P\left[\begin{array}{ccc}
-1.20 & -0.79 & 0 \\
0.60 & 0.15 & 0 \\
-3.30 & -2.10 & 1.00
\end{array}\right] \\
& +\left[\begin{array}{ccc}
54.45 & 34.65 & 0 \\
34.65 & 22.05 & 0 \\
0 & 0 & 0.10
\end{array}\right] .
\end{aligned}
$$

To solve this, we obtain

$$
\begin{aligned}
P & =\left[\begin{array}{ccc}
62.8002 & 39.9785 & -2.5095 \\
39.9785 & 25.4540 & -1.5962 \\
-2.5095 & -1.5962 & 0.8622
\end{array}\right] \\
F & =\left[\begin{array}{lll}
0.2406 & 0.2141 & 0.0140
\end{array}\right], \\
M_{c} & =\left[\begin{array}{ccc}
-0.8368 & -0.4668 & 0.0211 \\
1.1052 & 0.5995 & 0.0294 \\
-3.300 & -2.100 & 1.000
\end{array}\right] .
\end{aligned}
$$

Now, let the disturbance signal be

$$
w(k)= \begin{cases}0, & k<120 \\ 1, & 120 \leq k<160 \\ 0, & k>160 .\end{cases}
$$

For the following step reference signal, we perform MATLAB simulation results for the multirate controllers with preview action (let the preview length be $M_{d}=10$, i.e., $S_{d}=5$ ) and the one without preview action.

$$
r(k)= \begin{cases}0, & k<50 \\ 3, & k \geq 50 .\end{cases}
$$

The output responses of the linear discrete-time system in multirate output sampling are shown in Figure 1. The error signals are shown in Figure 2. We see from the figures that the preview action restrains the system disturbance effectively. And the simulation result shows better tracking in the case of disturbance preview action. Note that the error signal is asymptotically zero. Furthermore, the vibrations of output response curves are the multirate feature of this system.

\section{Conclusion}

This paper studies the disturbance preview optimal control problem for linear discrete-time systems in multirate output sampling. Instead of introducing a first-order difference operator we constructed the error system by the technique in [17],

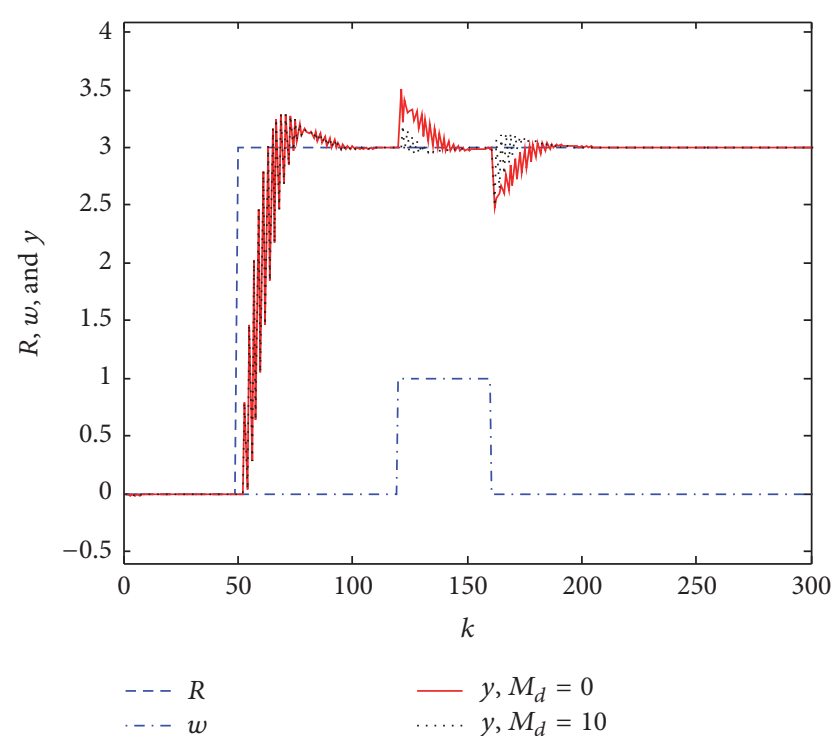

FIGURE 1: The output response of system (52) to step reference signal (58).

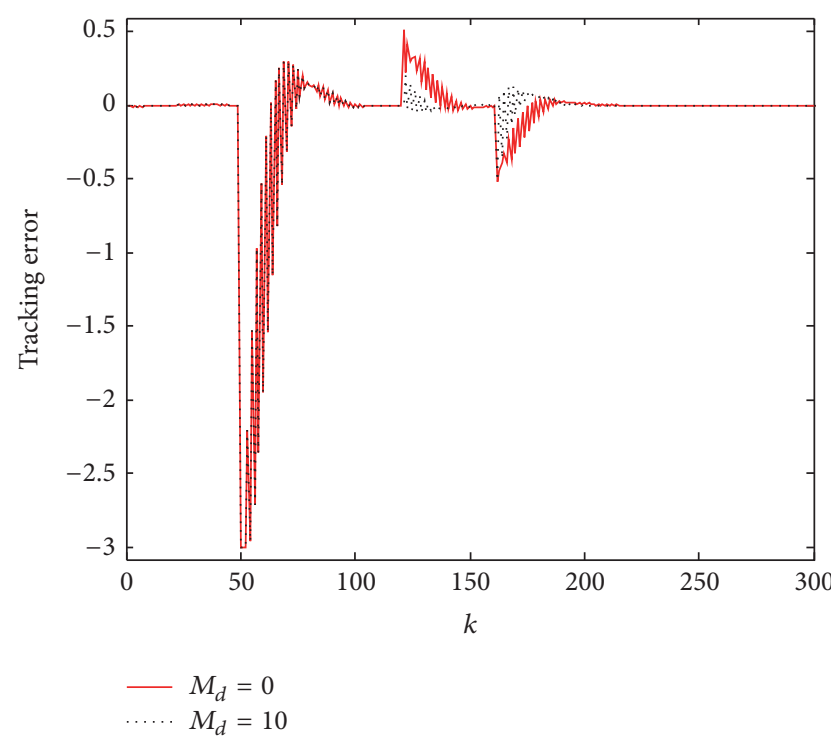

FIGURE 2: The tracking error of system (52) to step reference signal (58).

which made the process of constructing the augmented error system simpler. Then the multirate output control system was converted into a single-rate one by using the lifting scheme of this paper. We introduce the integral compensation in the process of constructing augmented error system, which can make the output track the reference signal and eliminate static error. Furthermore, we study a method that the highorder Riccati equation can be solved by solving a low-order Riccati equation. Finally, we obtain the preview controller for the original system. And the existence conditions of the controller were discussed. The numerical simulation showed the effectiveness of the proposed controller in this paper. 


\section{Competing Interests}

The authors declare no competing interests regarding the publication of this paper.

\section{Acknowledgments}

This work was supported by the Oriented Award Foundation for Science and Technological Innovation, Inner Mongolia Autonomous Region, China, and the National Natural Science Foundation of China (no. 61174209).

\section{References}

[1] J. Xiao, Multirate Sampling Digital Control System, Science Press, Beijing, China, 2003.

[2] T. Hagiwara and M. Araki, "Design of a stable state feedback controller based on the multirate sampling of the plant output," IEEE Transactions on Automatic Control, vol. 33, no. 9, pp. 812819, 1988.

[3] N. Satyanarayana and S. Janardhanan, "Multirate output sampling linear functional observer-based state feedback for systems with delay in state variables," International Journal of Modelling, Identification and Control, vol. 20, no. 1, pp. 47-55, 2013.

[4] A. P. Tiwari, G. D. Reddy, and B. Bandyopadhyay, "Design of periodic output feedback and fast output sampling based controllers for systems with slow and fast modes," Asian Journal of Control, vol. 14, no. 1, pp. 271-277, 2012.

[5] E. N. Mahmudov, Approximation and Optimization of Discrete and Differential Inclusions, Elsevier, 2011.

[6] T. Katayama, T. Ohki, T. Inoue, and T. Kato, "Design of an optimal controller for a discrete-time system subject to previewable demand," International Journal of Control, vol. 41, no. 3, pp. 677-699, 1985.

[7] T. Katayama and T. Hirono, "Design of an optimal servomechanism with preview action and its dual problem," International Journal of Control, vol. 45, no. 2, pp. 407-420, 1987.

[8] T. Tsuchiya and T. Egami, Digital Preview and Predictive Control, Beijing Science and Technology Press, Beijing, China, 1994, Translated by: F. Liao.

[9] N. Birla and A. Swarup, "Optimal preview control: a review," Optimal Control Applications \& Methods, vol. 36, no. 2, pp. 241268, 2015.

[10] E. Gershon and U. Shaked, " $H_{\infty}$ preview tracking control of retarded state-multiplicative stochastic systems," International Journal of Robust and Nonlinear Control, vol. 24, no. 15, pp. 21192135, 2014.

[11] Q. Zou and S. Devasia, "Precision preview-based stableinversion for nonlinear nonminimum-phase systems: the VTOL example," Automatica, vol. 43, no. 1, pp. 117-127, 2007.

[12] F. Liao, M. Cao, Z. Hu, and P. An, "Design of an optimal preview controller for linear discrete-time causal descriptor systems," International Journal of Control, vol. 85, no. 10, pp. 1616-1624, 2012.

[13] C. Gohrle, A. Schindler, A. Wagner, and O. Sawodny, "Design and vehicle implementation of preview active suspension controllers," IEEE Transactions on Control Systems Technology, vol. 22, no. 3, pp. 1135-1142, 2014.
[14] A. A. Ozdemir, P. Seiler, and G. J. Balas, "Design tradeoffs of wind turbine preview control," IEEE Transactions on Control Systems Technology, vol. 21, no. 4, pp. 1143-1154, 2013.

[15] C. M. Wang, X. M. Liu, and Y. J. Ji, Continuous and Discrete Control System, Science Press, Beijing, China, 2008.

[16] Y. Gu and M. Tomizuka, "Multi-rate feedforward tracking control for plants with nonminimum phase discrete time models," Journal of Dynamic Systems, Measurement and Control, vol. 123, no. 3, pp. 556-560, 1999.

[17] F. Liao, K. Takaba, T. Katayama, and J. Katsuura, "Design of an optimal preview servomechanism for discrete-time systems in a multirate setting," Dynamics of Continuous, Discrete \& Impulsive Systems_Series B: Applications \& Algorithms, vol. 10, no. 5, pp. 727-744, 2003.

[18] Q.-S. Shi and F.-C. Liao, "Design of an optimal preview controller for linear discrete-time multirate systems with statedelay," Journal of University of Science and Technology Beijing, vol. 33, no. 3, pp. 363-375, 2011.

[19] F. Liao, M. Tomizuka, M. Cao, and D. Wang, "Optimal preview control for discrete-time descriptor causal systems in a multirate setting," International Journal of Control, vol. 86, no. 5, pp. 844-854, 2013.

[20] D. Z. Zheng, Linear Systems Theory, Tsinghua University Press, Beijing, China, 2nd edition, 2002. 


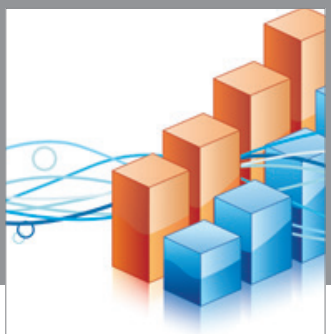

Advances in

Operations Research

vatem alat4

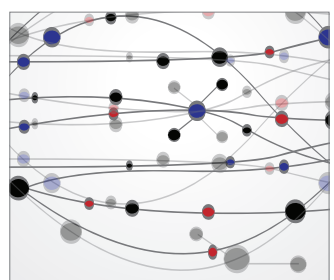

\section{The Scientific} World Journal
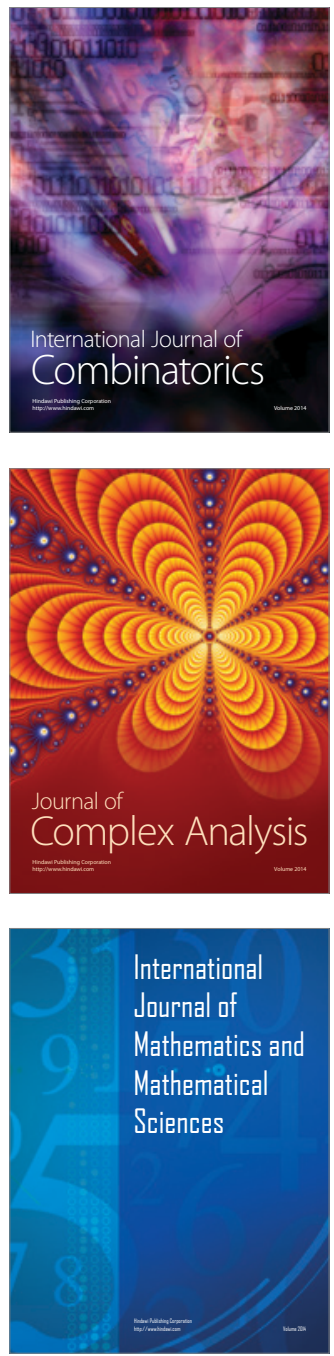
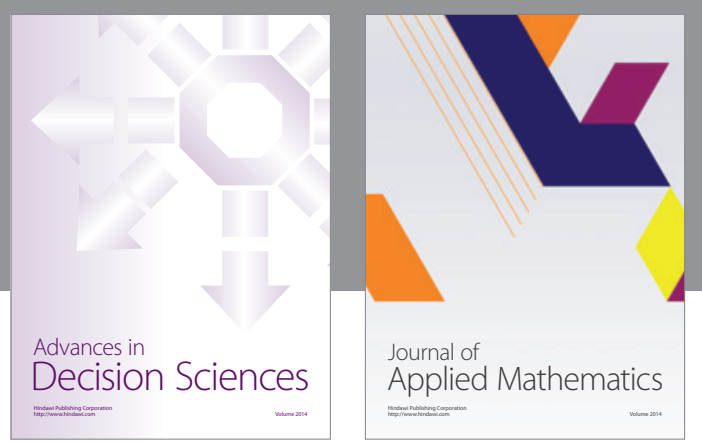

Algebra

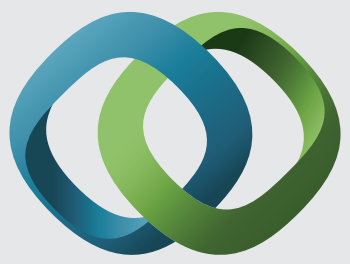

\section{Hindawi}

Submit your manuscripts at

http://www.hindawi.com
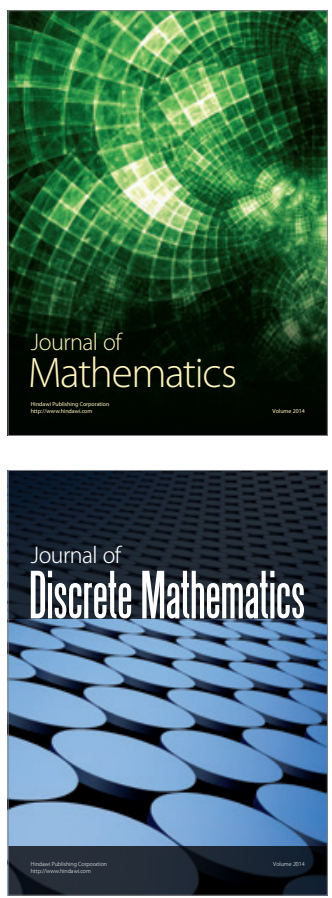

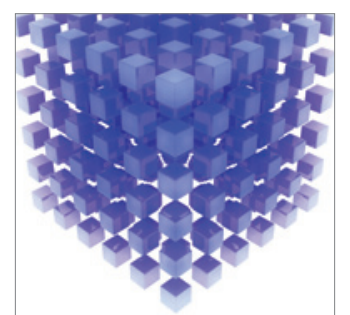

Mathematical Problems in Engineering
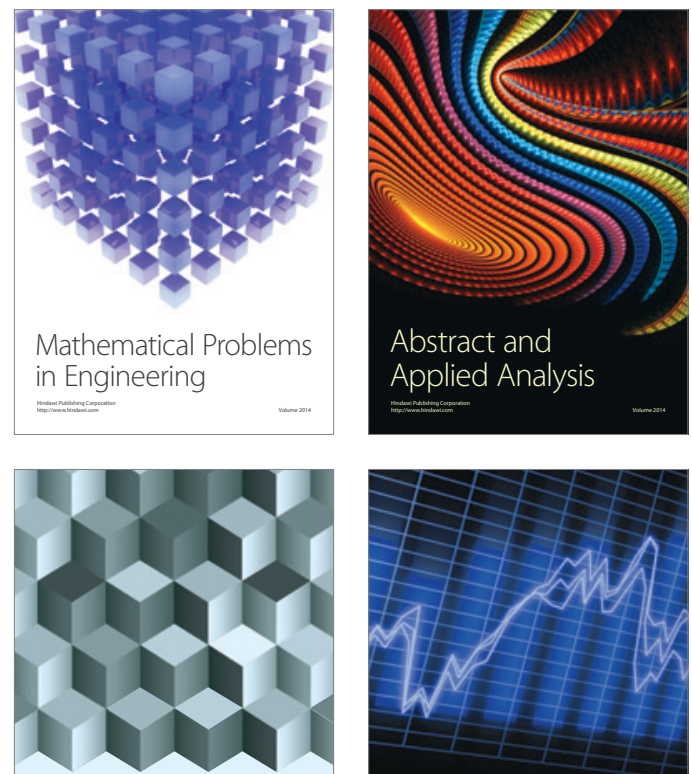

Journal of

Function Spaces

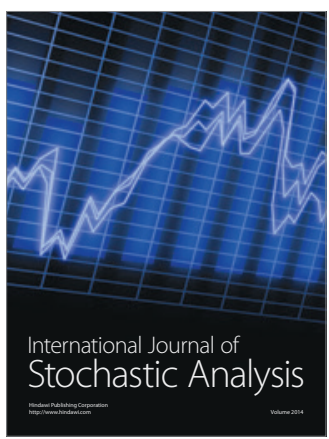

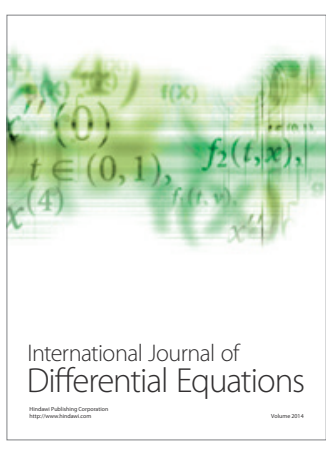
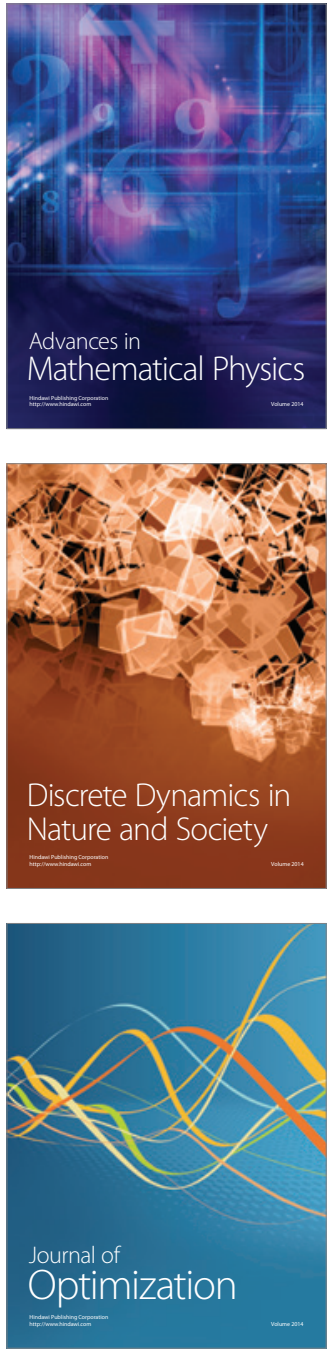